\title{
TIME DELAY OF RADIATION FROM PULSAR IN A BINARY SYSTEM THAT MOVES IN FIELD OF SCHWARZSCHILD BLACK HOLE
}

\author{
S. Komarov, A. Gorbatsievich, and A. Tarasenko \\ Department of Theoretical Physics, Belarusian State University, Nezavisimosti Ave., 4, 220030 Minsk, Belarus \\ E-mail: staskomarov@tut.by
}

Received 14 March 2017; revised 19 April 2017; accepted 20 June 2017

\begin{abstract}
A compact binary star that moves in a strong external gravitational field of a Schwarzschild black hole is considered. Decomposition of the redshift into a series with respect to the size of the binary system is obtained. This expression is used to calculate the redshift for a model binary system. Possible application of the results is discussed.
\end{abstract}

Keywords: redshift, time of arrival of pulsar pulses, supermassive black hole, general theory of relativity, reconstruction of motion

PACS: $04.25 . \mathrm{dg}$

\section{Introduction}

Recent observations of the Galactic centre $\left(\operatorname{Sgr~A}{ }^{*}\right)$ give evidences for existence of a supermassive black hole in this region with a mass of $\sim 4 \cdot 10^{6} M_{\odot}[1,2]$. Also they have provided an evidence for a large number of binary pulsars and stars in this region [3, 4 4 . In a volume of $1 \mathrm{pc}^{3}$ around Sgr $A^{\star}$ there are $\sim 10^{4}$ compact objects of about one stellar mass [5]. Presumably, about a half of these objects are bound in binary systems (NS-NS, NS-BH and BH-BH).

Studying the arrival time of the pulses that are emitted by a pulsar in a binary system gives possibility to test theories of gravity [6-10]. Also this data can be used to detect the gravitational radiation by its influence on the pulsar motion and on the propagation of the radiation to the observer (see, e.g. [11]). Electromagnetic radiation of a compact source in a gravitational field has been modelled in many papers (see, e.g. [12-17]). However, most of these approaches assume that the gravitational field is weak or that the radiation source is moving at a non-relativistic speed.
In [18] equations of motion of a compact binary system in a strong gravitational field have been obtained, and a method of calculation of the redshift of the light emitted by the binary system has been proposed.

In the present paper the approach described in [18] is used to calculate the next terms of the series expansion of the redshift, namely the terms that are quadratic in the size of the binary star $\rho$. The resulting expression is written in a covariant form. This expression contains quantities that are determined by the law of motion, and the function $z_{0}(\tau)$ that can be interpreted as the redshift of the source that is located at the center of mass of the binary star.

\section{Arrival time of the pulses}

Consider pulses that are emitted by a pulsar and are received by an observer. For pulsar moving in the neighbourhood of a supermassive black hole we describe the radiation in the geometrical optics approximation (see, e.g. [19]) (A typical wavelength of radiation used for observations $\lambda \lesssim 10^{3} \mathrm{~m}$ is much 
less than the scale of gravitational inhomogeneities $\mathrm{M} \sim 10^{9} \mathrm{~m}$ ). The observation time of a pulse can be calculated using the following expression:

$$
\begin{aligned}
& t_{\mathrm{TOA}}^{(N)}=t_{\mathrm{TOA}}^{(N-1)}+T_{\mathrm{p}}(z+1)=t_{\mathrm{TOA}}^{(N-1)} \\
& +T_{\mathrm{p}} \frac{\left(k^{i}\right)_{\mathrm{s}}\left(u_{i}\right)_{\mathrm{s}}}{\left(k^{i}\right)_{\mathrm{o}}\left(u_{i}\right)_{\mathrm{o}}} .
\end{aligned}
$$

Here $T_{\mathrm{p}}$ is the pulsar rotation period in the reference frame of the pulsar, $z$ is the redshift of the received light, $\left(u_{i}\right)_{s}$ and $\left(u_{i}\right)_{0}$ are the 4 -velocity of the source and the observer, respectively; $\left(k^{i}\right)_{s}$ and $\left(k^{i}\right)_{\mathrm{o}}$ are the 4-wave vectors at the point of emission and observation, respectively. $t_{\mathrm{TOA}}^{(N)}$ is the time of arrival of the $N$ th pulse and $t_{\mathrm{TOA}}^{(N-1)}$ is the time of arrival of the $(N-1)$ th pulse.

In this paper we consider the observer that is at rest at the spatial infinity. Generalizing the results to the case of a moving observer is not difficult (see, e.g. [18]).

Consider the vector field $k^{i}(x)$ which is defined at each point $x^{i}$ of the spacetime as the wave vector of the ray that is emitted at the point $x^{i}$ and is later received by the observer. $k^{i}$ is isotropic and satisfies the equation of geodesic:

$$
k^{i} k_{i}=0, k^{l} k_{i ; l}=0 .
$$

(In our notations a semicolon is the covariant derivative, a comma is the partial derivative.) Let $\zeta^{i}$ be a Killing vector $\left(\zeta_{i ; k}+\zeta_{k ; i}=0\right)$. Equations (2) imply that

$$
k^{i}\left(\zeta^{l} k_{l}\right),_{i}=0
$$

which means that $\zeta k_{l}$ is constant along the ray. If the spacetime is static, then $\partial / \partial t$ is the Killing vector and consequently $k_{4}=A=$ const. In an appropriate parametrization of the geodesic $A=-1$ and therefore $k_{4}=-1$ in the whole spacetime. It can be shown (see [18]) that for a static spherically-symmetric spacetime the vector field $k^{i}$ satisfies the following equation:

$$
k_{i, j}-k_{j ; i}=0
$$

The arrival time can be expressed as

$$
t_{\mathrm{TOA}}^{N}-t_{\mathrm{TOA}}^{N-1}=-T_{\mathrm{p}}\left(k^{i} u_{i}\right)_{\mathrm{s}} .
$$

Using this expression, the arrival time of pulses can be calculated given the redshift $z$ and the rotation period of the pulsar.

\section{Representation of the redshift of the source in the binary system}

The motion of a compact binary star in the external gravitational field can be described by the equations that are derived in [18]. These equations are obtained in a comoving reference frame by expanding the tidal forces into a series with respect to $\rho / r$, where $\rho$ is a characteristic size of the binary star, $r$ is a characteristic scale of the gravitational field (for example, the distance to the black hole). In the given paper a similar expansion of the redshift $z$ is obtained. Terms of the order $O\left(\rho^{2} / r^{2}\right)$ and $O(v / c)$ are kept in the series, where $v$ is a relative velocity of stars in the binary system.

Consider a radiation source moving along the world line $x_{P}^{i}\left(\tau_{P}\right)$, and the world line $\zeta^{i}(\tau)$, which is the world line of the center of mass. Here $\tau_{P}$ and $\tau$ are the proper time of the radiation source and the center of mass, respectively. Let the source be at the point $P$ at some moment of the time $\tau_{P}$. Consider the geodesic $x^{i}(\sigma)$ that starts at the point $P$ and crosses the world line of the center of mass orthogonally: $x^{i}(0)=\xi^{i}\left(\tau_{C}\right), x^{i}\left(\sigma_{P}\right)=x_{P}^{i}\left(\tau_{P}\right)$, where $\sigma$ is the geodesic distance. The intersection point will be denoted as $C, x_{C}^{i}=\xi^{i}\left(\tau_{C}\right)$ (see Fig.11). $x_{P}^{i}$ can be expressed as a Taylor series

$$
x_{P}^{i}=x_{C}^{i}+\frac{\mathrm{d} x^{i}(0)}{\mathrm{d} \sigma} \sigma_{P}+\frac{1}{2} \frac{\mathrm{d}^{2} x^{i}(0)}{\mathrm{d} \sigma^{2}} \sigma_{P}^{2}+O(\sigma)_{\mathrm{p}}^{3} .
$$

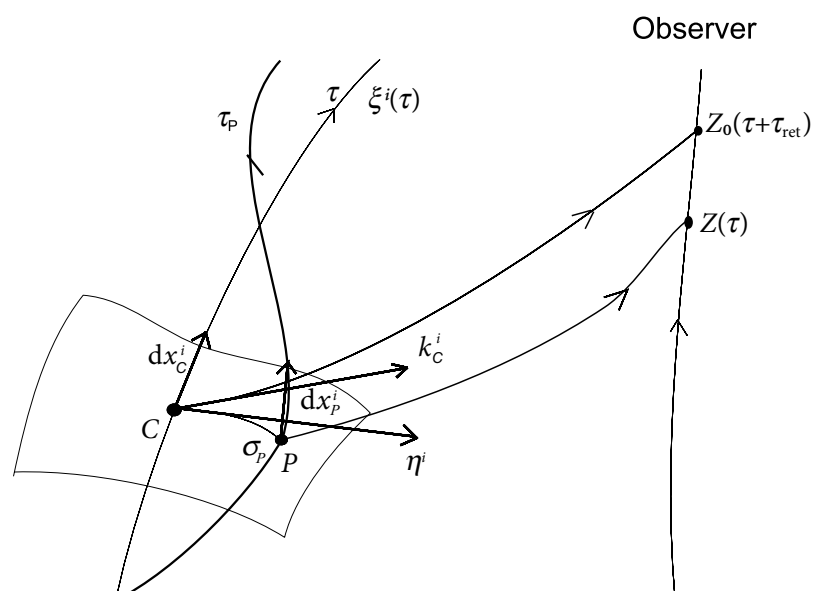

Fig. 1. To the derivation of the formula for the redshift. 
Here $\sigma_{P}$ is the geodesic distance from the point $P$ to $C$. Using the equation of geodesic and the orthogonality condition

$$
\begin{aligned}
& \frac{\mathrm{d}^{2} x^{i}}{\mathrm{~d} \sigma^{2}}+\Gamma_{j k}^{i} \frac{\mathrm{d} x^{j}}{\mathrm{~d} \sigma} \frac{\mathrm{d} x^{k}}{\mathrm{~d} \sigma}=0, \\
& g_{i j} \frac{\mathrm{d} \xi^{i}}{\mathrm{~d} \tau} \frac{\mathrm{d} x^{j}(0)}{\mathrm{d} \sigma}=0,
\end{aligned}
$$

series (6) can be transformed into

$$
x_{P}^{i}=x_{C}^{i}+\eta^{i} \sigma_{P}-\frac{1}{2} \Gamma_{j k}^{i}\left(x_{C}^{i}\right) \eta^{j} \eta^{k} \sigma_{P}^{2}+O\left(\sigma_{\mathrm{P}}^{3}\right) .
$$

Here $\Gamma_{j k}^{i}$ are the Christoffel symbols, $\eta^{i}=\mathrm{d} x^{i}(0) /$ $\mathrm{d} \sigma$. Also we will write $\mathrm{d} x_{C}^{i}$ as the differential of the coordinates in the direction of world line $\xi^{i}(\tau)$ in the point $C . \mathrm{d} x_{p}^{i}$ is the differential of coordinates in the direction of the world line of the source in the point $P$.

In order to find the series expansion of the 4-velocity $\left(u^{i}\right)_{s}$ and the 4 -wave vector $\left(k^{i}\right)_{s}$, consider the vector fields $k_{F}^{i}(\sigma)$ and $u_{F}^{i}(\sigma)$ that are covariantly constant along the geodesic $x^{i}(\sigma)$ and are equal to $\left(u^{i}\right)_{s}$ and $\left(k^{i}\right)_{s}$ at the point $P$ :

$$
\begin{gathered}
k_{F}^{i}\left(\sigma_{P}\right)=\left(k^{i}\right)_{s}, u_{F}^{i}\left(\sigma_{P}\right)=\left(u^{i}\right)_{s^{\prime}} \\
\frac{\mathrm{d} k_{F}^{i}(\sigma)}{\mathrm{d} \sigma}=-\Gamma_{j k}^{i} k_{F}^{j}(\sigma) \frac{\mathrm{d} x^{k}}{\mathrm{~d} \sigma}, \\
\frac{\mathrm{d} u_{F}^{i}(\sigma)}{\mathrm{d} \sigma}=-\Gamma_{j k}^{i} u_{F}^{j}(\sigma) \frac{\mathrm{d} x^{k}}{\mathrm{~d} \sigma} .
\end{gathered}
$$

Expanding the right-hand side of Eqs. (10) into series with respect to $\sigma_{p}$ yields

$$
\begin{aligned}
& \mathrm{d} k_{F}^{i}\left(\sigma_{P}\right)=-\Gamma_{j k}^{i} k_{F}^{j} \eta^{k}-\left(\Gamma_{j k, l}^{i} k_{F}^{j} \eta^{k} \eta^{l}\right. \\
& \mathrm{d} \sigma \\
& \left.-\Gamma_{j k}^{i} \Gamma_{l m}^{j} k_{F}^{l} \eta^{m} \eta^{k}-\Gamma_{j k}^{i} k_{F}^{j} \Gamma_{l m}^{k} \eta^{l} \eta^{m}\right) \sigma_{P}+O\left(\sigma_{\mathrm{P}}^{2}\right), \\
& \frac{\mathrm{d}^{2} k_{F}^{i}\left(\sigma_{P}\right)}{\mathrm{d} \sigma^{2}}=-\Gamma_{j k, l}^{i}{ }_{F}^{j} \eta^{k} \eta^{l} \\
& +\Gamma_{j k}^{i} \Gamma_{l m}^{j} k_{F}^{l} \eta^{m} \eta^{k}+\Gamma_{j k}^{i} k_{F}^{j} \Gamma_{l m}^{k} \eta^{l} \eta^{m}+O\left(\sigma_{\mathrm{P}}\right) .
\end{aligned}
$$

All quantities on the right-hand side of Eqs. (11) and (12) are calculated at the point $\sigma=0, x^{i}=x_{C}^{i}$. $k_{F}^{i}(0)$ can be written as a Taylor series:

$$
\begin{aligned}
& k_{F}^{i}(0)=k_{F}^{i}\left(\sigma_{P}\right)-\frac{\mathrm{d} k_{F}^{i}\left(\sigma_{P}\right)}{\mathrm{d} \sigma} \sigma_{P} \\
& +\frac{1}{2} \frac{\mathrm{d}^{2} k_{F}^{i}\left(\sigma_{P}\right)}{\mathrm{d} \sigma^{2}} \sigma_{P}^{2}+O\left(\sigma_{P}^{3}\right) .
\end{aligned}
$$

Since $\left(k^{i}\right)_{s}=k^{i}\left(x_{P}^{i}\right)=k_{F}^{i}\left(\sigma_{P}\right)$, it is also given by

$$
\begin{aligned}
& k_{F}^{i}\left(\sigma_{P}\right)=k^{i}\left(x_{C}^{i}\right)+k_{j}^{i},\left(x_{C}^{i}\right) \eta^{j} \sigma_{P} \\
& +\frac{1}{2} k_{,{ }_{l, m}}^{i}\left(x_{C}^{i}\right) \eta^{l} \eta^{m} \sigma_{P}^{2}+O\left(\sigma_{P}^{3}\right) .
\end{aligned}
$$

Substituting Eqs. (11), (12) and (14) into Eq. (13) yields

$$
\begin{aligned}
& k_{F}^{i}(0)=k^{i}\left(x_{C}^{i}\right)+\frac{\mathrm{D}}{\mathrm{D} \delta} k^{i}\left(x_{C}^{i}\right) \sigma P \\
& +\frac{1^{2}}{2} \frac{\mathrm{D}^{2}}{\mathrm{D} \delta^{2}} k^{i}\left(x_{C}^{i}\right) \sigma_{P}^{2} .
\end{aligned}
$$

Here $\frac{\mathrm{D}}{\mathrm{D} \delta}$ denotes the covariant derivative in the direction of $\eta^{i}$.

Using Eq. (8) the following expression for the 4-velocity can be obtained:

$$
\begin{aligned}
& \left(u^{i}\right)_{s}=u_{F}^{i}(P)=\frac{\mathrm{d} x_{P}^{i}}{\mathrm{~d} \tau_{P}}=\frac{\mathrm{d} \tau}{\mathrm{d} \tau_{P}}\left[u_{C}^{i}\right. \\
& +\frac{\mathrm{d}}{\mathrm{d} \tau}\left(\eta^{i} \sigma_{P}\right)-\frac{1}{2} \Gamma_{j k, l}^{i} u_{C}^{l} \eta^{j} \eta^{k} \sigma_{P}^{2} \\
& \left.-\Gamma_{j k}^{i} \frac{\mathrm{d}\left(\eta^{j} \sigma_{P}\right)}{\mathrm{d} \tau} \eta^{k} \sigma_{P}\right]+O\left(\sigma_{P}^{3}, \sigma_{P} v\right),
\end{aligned}
$$

where $u_{C}^{i}=\frac{\mathrm{d} x_{C}^{i}}{\mathrm{~d} \tau}$ is the 4 -velocity of the centre of mass. Expanding $u_{F}^{i}$ into a series analogically to Eq. (13) results in the following expression:

$$
\begin{aligned}
& u_{F}^{i}(0)=\frac{\mathrm{d} \tau}{\mathrm{d} \tau_{P}}\left[u_{C}^{i}+\frac{\mathrm{D}}{\mathrm{D} \tau}\left(\eta^{i} \sigma_{P}\right)\right. \\
& \left.+\frac{1}{2} R_{m s}^{i} u_{C}^{l} \eta^{m} \eta^{s} \sigma_{P}^{2}\right]+O\left(\sigma_{P}^{3}, \sigma_{P} v\right)
\end{aligned}
$$

Here all quantities are calculated at the point $\sigma=0$, $x^{i}=x_{C}^{i}$. Since the fields $g_{i j}, u_{F}^{i}$ and $k_{F}^{j}$ are parallelly transported along the line $x^{i}(\sigma)$, and a scalar product of vectors does not change during the parallel transportation,

$$
\left(g_{i j} k^{i} u^{j}\right)_{s}=g_{i j}\left(x_{C}^{i}\right) k_{F}^{i}(0) u_{F}^{j}(0) .
$$


Using Eqs. (5), (15), (17) and (18) we obtain

$$
\begin{aligned}
& 1+z=-\frac{\mathrm{d} \tau}{\mathrm{d} \tau_{P}}\left[k_{i} u_{C}^{i}+\frac{\mathrm{d}}{\mathrm{d} \tau}\left(k_{i} \eta^{i} \sigma_{P}\right)\right. \\
& \left.+\frac{1}{2} \frac{\mathrm{d}}{\mathrm{d} \tau}\left(k_{m ; s} \eta^{m} \eta^{s} \sigma_{P}^{2}\right)\right]+O\left(\sigma_{P}^{3}, \sigma_{P} v, v^{2}\right) .
\end{aligned}
$$

Let $\mathrm{d} \tau$ and $\mathrm{d} \tau_{P}$ be the proper time intervals of the centre of mass and the radiation source, respectively. These quantities are given by

$$
\begin{aligned}
& \mathrm{d} \tau^{2}=g_{i j}\left(x_{C}^{l}\right) \mathrm{d} x_{C}^{i} \mathrm{~d} x_{C}^{j}, \\
& \mathrm{~d} \tau_{\mathrm{P}}^{2}=g_{i j}\left(x_{P}^{l}\right) \mathrm{d} x_{P}^{i} \mathrm{~d} x_{P}^{j} .
\end{aligned}
$$

Expanding (20) into a series results in

$$
\frac{\mathrm{d} \tau}{\mathrm{d} \tau_{P}}=1-R_{l i j m} u_{C}^{i} u_{C}^{j} \eta^{l} \eta^{m} \sigma_{P}^{2}+O\left(\sigma_{P}^{3}, \sigma_{P} v, v^{2}\right) .
$$

For a given moment of time the observer receives rays with the following redshift:

$$
\begin{aligned}
& 1+z_{0}(\tau) \equiv-k_{i}(C) \frac{\mathrm{d} x_{C}^{i}\left(\tau-\tau_{\mathrm{ret}}\right)}{\mathrm{d} \tau}, \\
& 1+z(\tau) \equiv-k_{i}(P) \frac{\mathrm{d} x_{P}^{i}(\tau)}{\mathrm{d} \tau_{P}}
\end{aligned}
$$

Here $\tau_{\text {ret }}$ is the delay of the proper time of emission. $\tau_{\text {ret }}$ can be calculated using the requirement that the arrival time of the rays originating at the points $C$ and $P$ is the same. Using this definition we rewrite Eq. (19):

$$
\begin{aligned}
& 1+z(\tau)=\frac{\mathrm{d} \tau}{\mathrm{d} \tau_{P}}\left[1+z_{0}\left(\tau+\tau_{\mathrm{ret}}\right)-\frac{\mathrm{d}}{\mathrm{d} \tau}\left(k_{i} \eta^{i} \sigma_{P}\right)\right. \\
& \left.-\frac{1}{2} \frac{\mathrm{d}}{\mathrm{d} \tau}\left(k_{m ; s} \eta^{m} \eta^{s} \sigma_{P}^{2}\right)\right]+O\left(\sigma_{P}^{3}, \sigma_{P} v, v^{2}\right) .
\end{aligned}
$$

(For convenience we do not write the argument $\tau$ of the functions on the right-hand side.)

For the calculation of $\tau_{\text {ret }}$ we use the following general equation that holds for an isotropic geodesic (see, e.g. [20]):

$$
k_{i} \mathrm{~d} x^{i}=\text { const (along an isotropic geodesic). }
$$

Here $\mathrm{d} x^{i}$ is the coordinate distance between two close geodesics. Applying this equation to the points $C$ and $P$ yields

$$
k_{i} \frac{\mathrm{d} x^{i}}{\mathrm{~d} \sigma} \mathrm{d} \sigma=-\left(k_{i} u^{i}\right)_{o} \mathrm{~d} t=\mathrm{d} t .
$$

Consequently, we receive

$$
\begin{aligned}
& t_{\text {ret }}=\int_{0}^{\sigma_{P}} k_{i} \frac{\mathrm{d} x^{i}}{\mathrm{~d} \sigma} \mathrm{d} \sigma \\
& \approx k_{i} \eta^{i} \sigma_{P}+\frac{1}{2} k_{i ; j} \eta^{i} \eta^{j} \sigma_{P}^{2} .
\end{aligned}
$$

$\tau_{\text {ret }}$ can be expressed as

$$
\begin{aligned}
& \tau_{\text {ret }}=\int_{0}^{t_{\mathrm{ret}}} \mathrm{d} t z+1 \approx \frac{k_{i} \eta^{i}}{z_{0}+1} \sigma_{P} \\
& +\frac{1}{2} \frac{k_{i ; j} \eta^{i} \eta^{j}}{z_{0}+1} \sigma_{P}^{2}-\frac{1}{2} \frac{\left(k_{i} \eta^{i}\right)^{2}}{\left(z_{0}+1\right)^{3}} \frac{\mathrm{d} z_{0}}{\mathrm{~d} \tau} \sigma_{P}^{2} .
\end{aligned}
$$

Substituting Eqs. (21) and (27) into Eq. (23) and denoting $n^{i} \equiv k^{i} /\left(1+z_{0}\right)$ we obtain

$$
\begin{aligned}
& 1+z=\left(1+z_{0}\right)\left[1-\frac{\mathrm{d}}{\mathrm{d} \tau}\left(n_{i} \eta^{i} \sigma_{P}\right)-\frac{1}{2} \frac{\mathrm{d}}{\mathrm{d} \tau}\left(n_{i ; j} \eta^{i} \eta^{j} \sigma_{P}^{2}\right)\right. \\
& +\frac{1}{2}\left(n_{i} \eta^{i}\right)^{2} \frac{\mathrm{d}}{\mathrm{d} \tau}\left(\frac{1}{z_{0}+1} \frac{\mathrm{d} z_{0}}{\mathrm{~d} \tau}\right) \sigma_{P}^{2} \\
& \left.-R_{l i j m} u_{C}^{i} u_{C}^{i} \eta^{l} \eta^{m} \sigma_{P}^{2}\right]+O\left(\sigma_{P}^{3}, \sigma_{P} v, v^{2}\right)
\end{aligned}
$$

where all quantities are calculated at the proper time $\tau$.

In order to find the observation time of the ray it is necessary to calculate the following integral:

$$
t=\int_{0}^{\tau_{P}}\left(1+z\left(\tau^{\prime}\right)\right) \mathrm{d} \tau^{\prime} .
$$

The redshift as a function of the observation time can be calculated using formulas (28) and (29). An example of a redshift as a function of the proper time is shown in Fig. 2 .

Equations of motion of a binary system in the external gravitational field are considered in [18]. The solution of these equations allow one to find the Fermi coordinates of the source $X^{(\alpha)}\left(\tau_{P}\right)$ as a function of the proper time. These quantities are related to the notations used in the present paper:

$$
n_{i} \eta^{i} \sigma_{P}=n_{(\alpha)} X^{(\alpha)}
$$




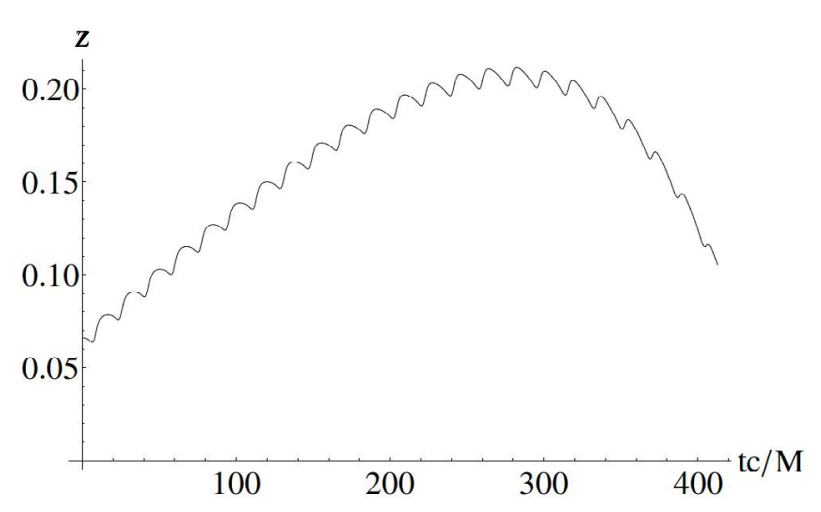

Fig. 2. Redshift as a function of the observation time

\section{Consequently,}

$$
n_{i, j} \eta^{i} \eta^{j} \sigma_{P}^{2}=\frac{\mathrm{d}}{\mathrm{d} \sigma}\left(n_{(\alpha)} X^{(\alpha)}\right) \sigma_{P},
$$

where $n^{(\alpha)}$ is the unit vector of the ray in Fermi coordinates: $n_{(\alpha)} n^{(\alpha)}=1$.

In the present paper an expression for the redshift of a radiation source that moves in the external Schwarzschild field has been obtained. Expression (28) with minor changes can be applied not only to the Schwarzschild field but also to any gravitational field, for example, the field of a Kerr black hole or an external gravitational wave.

The obtained expression for the redshift and the method of its calculation can be applied to a binary pulsar in the neighbourhood of the Galactic centre. A more interesting problem is the reconstruction of the motion of such binary system by its redshift. A similar problem has been considered for the case when extra images of the source are visible (see [21]). We are planning to leave the inverse problem for a separate paper.

\section{References}

[1] F.K. Baganoff, M.W. Bautz, W.N. Brandt, G. Chartas, E.D. Feigelson, G.P. Garmire, Y. Maeda, M. Morris, G.R. Ricker, L.K. Townsley, and F. Walter, Rapid X-ray flaring from the direction of the supermassive black hole at the Galactic Centre, Nature 413, 45-48 (2001), http://dx.doi. org/10.1038/35092510

[2] M.R. Morris, The environment of the Galaxy's central black hole, in: The Galactic Black Hole. Lectures on General Relativity and Astrophysics, eds. H.F. Falcke and F.W. Hehl (IOP Publishing Ltd, 2003) pp. 95-122.

[3] K. Perez, C.J. Hailey, F.E. Bauer, R.A. Krivonos, K. Mori, F.K. Baganoff, N.M. Barrière, S.E. Boggs, F.E. Christensen, W.W. Craig, et al., Extended hard-X-ray emission in the inner few parsecs of the Galaxy, Nature 520, 646-649 (2015), http:// dx.doi.org/10.1038/nature14353

[4] S. Gillessen, F. Eisenhauer, S. Trippe, T. Alexander, R. Genzel, F. Martins, and T. Ott, Monitoring stellar orbits around the Massive Black Hole in the Galactic Center, Astrophys. J 692, 10751109 (2009), http://dx.doi.org/10.1088/0004637X/692/2/1075

[5] V.E. Zavlin, XMM-Newton observations of four millisecond pulsars, Astrophys. J. 638, 951-962 (2006), http://dx.doi.org/10.1086/449308

[6] R. Blandford and S.A. Teukolsky, Arrival-time analysis for a pulsar in a binary system, Astrophys. J. 205, 580-591 (1976), http://dx.doi. org/10.1086/154315

[7] J.W. Weisberg and J.H. Taylor, Relativistic binary pulsar B1913+16: Thirty years of observations and analysis, in: Binary Radio Pulsars, ASP Conference Series, eds. F.A. Rasio and I.H. Stairs (2004), arXiv:astro-ph/0407149

[8] N. Wex, Testing relativistic gravity with radio pulsars, arXiv:1402.5594 (2013), https://arxiv.org/ $\mathrm{abs} / 1402.5594$

[9] R.T. Edwards, G.B. Hobbs, and R.N. Manchester, Tempo2, a new pulsar timing package. II. The timing model and precision estimates, Mon. Not. R. Astron. Soc. 372, 1549-1574 (2006), https://arxiv. org/abs/astro-ph/0607664

[10]T. Damour and N. Deruelle, General relativistic celestial mechanics of binary systems. II. The postNewtonian timing formula, Ann. Inst. Henri Poincaré 44, 263-292 (1986), http://www.numdam.org/item?id=AIHPA_1986_44_3_263_0

[11]M.A. McLaughlin, The North American Nanohertz Observatory for Gravitational Waves, arXiv:1310.0758v1 (2013), https://arxiv.org/ abs/0909.1058

[12]Y. Wang, F.A. Jenet, T. Creighton, and R.H. Price, Strong field effects on pulsar arrival times: circular orbits and equatorial beams, Astrophys. J. 697, 
237-246 (2008), http://dx.doi.org/10.1088/0004$637 \mathrm{X} / 697 / 1 / 237$

[13]Y. Wang, T. Creighton, R.H. Price, and F.A. Jenet, Strong field effects on pulsar arrival times: general orientations, Astrophys. J. 705, 12521259 (2009), http://dx.doi.org/10.1088/0004$637 \mathrm{X} / 705 / 2 / 1252$

[14]K. Stovall, T. Creighton, R.H. Price, and F.A. Jenet, Observability of pulsar beam bending by the Sgr $\sim A^{*}$ black hole, Astrophys. J. 744, 143150 (2012), http://dx.doi.org/10.1088/0004637X/744/2/143

[15] R. Angelil, P. Saha, and D. Merritt, Toward relativistic orbit fitting of Galactic center stars and pulsars, Astrophys. J. 720, 1303-1310 2010, http:// dx.doi.org/10.1088/0004-637X/720/2/1303

[16]K. Liu, N. Wex, M. Kramer, J.M. Cordes, and T.J.W. Lazio, Prospects for probing the spacetime of Sgr $A^{\star}$ with pulsars, Astrophys. J. 747, 11-23 (2012), http://dx.doi.org/10.1088/0004$637 \mathrm{X} / 747 / 1 / 1$
[17]F. Zhang, Y. Lu, and Q. Yu, On testing the Kerr metric of the massive black hole in the Galactic center via stellar orbital motion: full general relativistic treatment, Astrophys. J. 809, 127 (2015), http://dx.doi.org/10.1088/0004$637 \mathrm{X} / 809 / 2 / 127$

[18]A. Gorbatsievich, S. Komarov, and A. Tarasenko, Optical appearance of a compact binary system in the neighbourhood of supermassive black hole, arXiv:1702.08381 (2017), https://arxiv.org/ abs/1702.08381

[19]H. Stephani, Relativity. An Introduction to Special and General Relativity, 3rd ed. (Cambridge University Press, 2004).

[20]J. Sing, Relativity: The General Theory (NorthHolland Publishing Company, Amsterdam, 1960).

[21]A. Tarasenko, Reconstruction of a compact object motion in the vicinity of a black hole by its electromagnetic radiation, Phys. Rev. D 81, 123005 (2010), http://dx.doi.org/10.1103/PhysRevD.8.

\title{
DVINARĖS SISTEMOS PULSARO, JUDANČIO ŠVARCŠILDO JUODOSIOS BEDUGNĖS LAUKE, SPINDULIUOTĖS ATSILIKIMAS
}

\author{
S. Komarov, A. Gorbatsievich, A. Tarasenko \\ Baltarusijos valstybinis universitetas, Minskas, Baltarusija
}

